\title{
UAV Interference to Existing Satellite Services in C-band
}

\author{
Evgenii Vinogradov \\ Achiel Colpaert \\ Sofie Pollin \\ evgenii.vinogradov@kuleuven.be \\ achiel.colpaert@kuleuven.be \\ sofie.pollin@kuleuven.be \\ KU Leuven, Department of Electrical Engineering - ESAT \\ Leuven, Belgium
}

\begin{abstract}
Cellular connected UAVs can operate in beyond visual line of sight scenarios. However, inter-cell interference has been shown to be the main limiting factor at high altitudes, so that many companies and researchers try to use less congested parts of the radio spectrum. One of the pioneer bands identified in Europe for the early deployment of $5 \mathrm{G}$ systems is the $3400-3800 \mathrm{MHz}$ (C-band), which is still relatively free. However, this band is already used in Europe (and elsewhere) for Fixed Satellite Service (FSS). Coexistence with broadband mobile services should be investigated to properly identify operational conditions in real environments. Very Small Aperture Terminals (VSATs) represent an important class of FSS terminals worldwide. Unlike the large Earth stations, they use smaller antennas. VSATs do not require licensing (i.e. their locations are unknown). Moreover, they typically have modest ability for mitigating interference due to the relatively cheap consumer-grade equipment being used. Coexistence of cellular connected UAVs and FSS is not investigated in literature. In this article, we provide useful information to regulatory bodies for defining appropriate rules for coexistence between FSS and UAVs connected to cellular networks. Using a realistic 3D simulator model of a city combined with the state of the art channel models, this study confirms that UAVs create significant interference to FSS. We estimated on- and off-axis separation distances as well as the permissible interference levels allowing functioning of FSS. We defined the minimum separation distances and angles for different interference types (long- and short- term interference).
\end{abstract}

\section{CCS CONCEPTS}

- Computer systems organization $\rightarrow$ Embedded systems; $R e$ dundancy; Robotics; • Networks $\rightarrow$ Network reliability.

\section{KEYWORDS}

UAV; 5G; sub-6 GHz; interference; VSAT; satellite; FSS; coexistence

Permission to make digital or hard copies of all or part of this work for personal or classroom use is granted without fee provided that copies are not made or distributed for profit or commercial advantage and that copies bear this notice and the full citation on the first page. Copyrights for components of this work owned by others than the author(s) must be honored. Abstracting with credit is permitted. To copy otherwise, or republish, to post on servers or to redistribute to lists, requires prior specific permission and/or a fee. Request permissions from permissions@acm.org.

DroNet'19, June 21, 2019, Seoul, Republic of Korea

(c) 2019 Copyright held by the owner/author(s). Publication rights licensed to ACM ACM ISBN 978-1-4503-6772-1/19/06 . \$ \$15.00

https://doi.org/10.1145/3325421.3329767
ACM Reference Format:

Evgenii Vinogradov, Achiel Colpaert, and Sofie Pollin. 2019. UAV Interference to Existing Satellite Services in C-band. In The 5th Workshop on Micro Aerial Vehicle Networks, Systems, and Applications (DroNet'19), June 21, 2019, Seoul, Republic of Korea. ACM, New York, NY, USA, 6 pages. https://doi.org/10.1145/3325421.3329767

\section{INTRODUCTION}

In recent years, unmanned aerial vehicles (UAVs) have attracted a lot of research and industry attention driven by the promise of many applications [19]. Many of these applications consider the $\mathrm{UAV}$ to be connected to a $4 \mathrm{G}$ or $5 \mathrm{G}$ telecommunications network. Currently deployed cellular networks are unable to deliver a reliable connectivity due to inter-cell interference[17]. This is the reason why some less congested parts of the spectrum (e.g. 4G and 5G 3.5 GHz band) are foreseen to be used for future UAV applications. This requires the determination of the conditions for coexistence with the satellite systems operating in C-band (3.5-4.2 GHz). One of the services that might be affected is television broadcasting.

The first kind of equipment that might be affected are large Earth station sites at known locations. In this case, coordination contours can be defined as in [10]. Moreover, these stations usually have complex interference protection mechanisms, so that the interference influence on the service is limited. The second type of equipment are the Very Small Aperture Terminals (VSAT, 0.6-2.4 m diameter antenna dishes). A VSAT is consumer-grade receive-only equipment for TV reception (sometimes it can include a transmitter to enable internet access). The coordination between the cellular network agents (Base Stations and users) and VSAT stations is impossible, due to the lack of information about the geographical location or the operation frequency of the satellite TV receivers (VSATs do not require licensing). Moreover, unlike the large Earth stations, VSATs are typically not protected from possible interference from other systems.

Interference from mobile broadband services to C-band satellite Earth station receivers was considered in several studies [6, 8$10,12]$. However, works [8-10, 12] considered the terminals used for bi-directional communications, and having antenna diameters of larger than $4.5 \mathrm{~m}$. Authors of [6] reported trial results for Long Term Evolution (LTE) base stations (eNb) co-channel and adjacentchannel interference to consumer VSAT TV receivers. The minimum distance of $1.2 \mathrm{~km}$ between the interfering $\mathrm{eNb}$ and the victim VSAT receiver was determined. [8] presents a study of the impact of mobile broadband services into FSS receivers. This study was 
based on the determination of mitigation zones which are defined as a geographical area, within which there is a potential for harmful interference to the FSS. They also state that these distances with respect to the FSS depend on the characteristics of the eNb. A decision by administration on the deployment of an eNb should take into account the FSS usage of the band. In [9], the authors add more details to the preceding report by stating that co-existence of the mobile broadband services and the FSS in the same band can be successful through coordination on a case-by-case basis. Their results show that the interference caused by ground user equipment (UE) is less than that of eNb, thus separation that prevents interference from eNbs will also protect FSS from UE interference. In the results they consider only macrocells as micro, pico and femfto cells will result in considerably lower separation distances due to lower power and shielding by houses in the vicinity. In a more recent report [10], operational guidelines on spectrum sharing between mobile broadband services and the existing FSS in the 3.6-3.8 GHz band are presented. They suggest two approaches, specifying the maximum permitted interference powers at the FSS receivers and allowing the mobile operators flexibility to comply to these limits. A second approach of specifying explicit restrictions on the the frequency or location or the Equivalent Isotropically Radiated Power of the eNb. These restrictions can be expressed in terms of exclusion and/or restriction zones. Note that only interference from elevated eNbs and ground user equipment nodes was investigated in literature.

Due to the higher probability of having LOS links between VSATs and UAVs[19], one can expect the interference levels from UAVs to be significantly higher than in the case of ground-level UE. Note that UAV-mounted equipment was never considered in the literature. In this paper, we simulate 3D interference created by a UAV-mounted UE to the VSAT receivers of satellite TV. It is the first time in literature that the co-existence of UAVs and satellite broadcasting services is investigated.

Performing measurements is indeed the most precise way of interference estimation, however, due to the limited number of trials possible, this approach gives only a fragmented picture of the situation and it is impossible to generalize the obtained results for the great variation of propagation paths and all potential scenarios. For this reason, this research is based on large-scale simulation campaign taking advantage of a semi-deterministic channel simulator based on real 3D city maps. This approach gives a reliable evaluation of the interference levels and, consequently, the minimum separation distance needed for error-free operation of satellite services in C-band.

The paper is structured as follows: in Section 2, we briefly describe the simulator and the interference estimation approach; the relevant results are reported in Section 3. Finally, Section 4 draws the main conclusions of the paper.

\section{SYSTEM MODEL}

This section describes the system model that is used to simulate the interference created by UAVs to existing satellite services. Out of all 5G NR operating bands specified in [4], band n77 is chosen, specifying a frequency band of 3.3-4.2 GHz. In this article, we consider $B=10 \mathrm{MHz}$, as this is most common used in practise. The simulations focus on interference experienced by the VSAT from

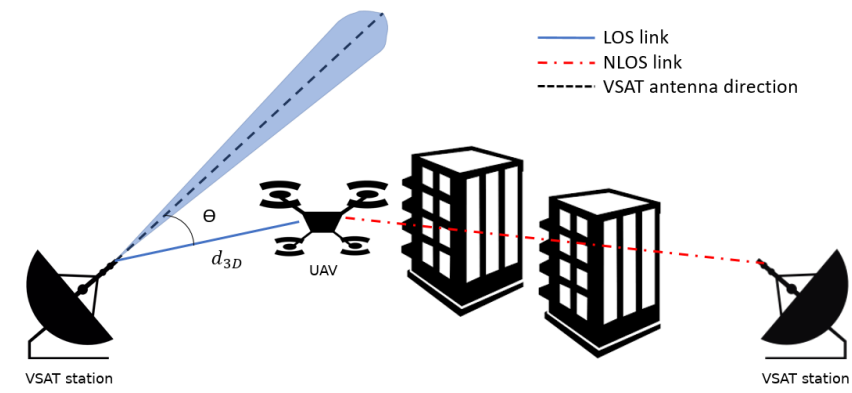

Figure 1: Graphic representation of the considered system

the uplink connection (i.e., from UAV to eNb or $5 \mathrm{G} \mathrm{gNb}$ ) of UAVs flying up to an altitude of $100 \mathrm{~m}$ above ground level (AGL). The interference levels are expressed in terms of the offset angle $\Theta$ and $3 \mathrm{D}$ distance $d_{3 D}$ separating the nodes (see Figure 1).

\subsection{Setup}

To study the influence of UAV interference on the VSAT performance, the simulator combining channel models with a 3D map of a real city presented in [7] has been extended to include the uplink communication in $3.5 \mathrm{GHz}$ frequency band. Table 1 contains the main simulation parameters.

Table 1: Simulation parameters.

\begin{tabular}{lc}
\hline \multicolumn{1}{c}{ Parameters } & Values \\
\hline Carrier frequency & $3.5 \mathrm{GHz}$ \\
Signal bandwidth, B & $10 \mathrm{MHz}$ \\
UAV Tx power, $P_{U A V}$ & $23 \mathrm{dBm}$ \\
\# UAV positions (per VSAT) & $150 \times 150 \times 18$ \\
VSAT diameter, $D$ & $1.8 \mathrm{~m}$ \\
VSAT max antenna gain, $G_{0}$ & $35.7 \mathrm{dBi}$ \\
$\phi_{\text {min }}$ & $2.5 \mathrm{deg}$ \\
\# VSAT & 100 \\
\# satellites & 5 \\
Noise temperature of receiving system,$T_{r}$ & $100 \mathrm{~K}[13]$ \\
\hline
\end{tabular}

2.1.1 Environment. All simulations were performed in a real 3D environment. The 3D surface scan with $1 \mathrm{~m}$ resolution of Flanders, Belgium [1] was used. The city of Ghent was chosen for the simulations, since it represents a typical European city. The environment can be categorized as sub-urban or urban.

VSATs are located randomly in the city, outside buildings at a height of $10 \mathrm{~m}$ AGL. An area of $3000 \mathrm{~m}$ by $3000 \mathrm{~m}$ is used for the analysis.

For each VSAT location, a single UAV carrying a UE is subsequently positioned on a grid with resolution of $20 \mathrm{~m}$. The considered altitudes $h_{U A V}$ are ranging from $h_{V S A T}$ to $h_{V S A T}+90 \mathrm{~m}$ with $5 \mathrm{~m}$ resolution. The distance between the nodes is ranging between $5 \mathrm{~m}$ to $\approx 4000 \mathrm{~m}$ depending on the VSAT location. The total number of analyzed interference realizations is around $186 \cdot 10^{6}\left(37.3 \cdot 10^{6} \mathrm{UAV}\right.$ positions per VSAT deployment, 5 different satellites). Next, the power received by VSAT is calculated as shown in the following subsection. 
2.1.2 Link Model. This subsection explains all the components out of which the link model consists. The resulting interference experienced by VSAT can be calculated in $\mathrm{dB}$ as:

$$
I=P_{U A V}+G_{U A V}+G_{V S A T}-P L,
$$

where $P L$ is the distance and environment-dependent channel attenuation (path loss), $G_{U A V}$ and $G_{V S A T}$ are the antenna gains of UAV and VSAT, respectively, values can be found in table 1.

Antenna patterns: VSATs are equipped with antennas with 3D radiation patterns modeled as APEREC026V01 antenna pattern in [15] and the gain for the main lobe is modeled according to the extensions given in [14]. The antenna parameters are given in Table 1 . The elevation and azimuth angles are chosen according to the targeted satellites (see Table 2).

For the aerial user equipment (AUE) mounted on a UAV, an omnidirectional antenna with maximum gain of $2.15 \mathrm{dBi}$ was used (modeled as in [16]).

Line of sight: When checking whether a UAV location is in Lineof-sight (LOS) with the VSAT, a line is drawn between the nodes in the $3 \mathrm{D}$ environment. If there are no intersections with the 3D environment the link between VSAT and UAV is considered LOS; the link is considered non-line of sight (NLOS) if obstructions do exist. An illustration can be seen in Figure 1.

Path loss model: The path loss (PL) model for $3.5 \mathrm{GHz}$ is defined by combining the work in [3], which specifies an cellular path loss model for ground users up to $22.5 \mathrm{~m}$ AGL, and the work in [2] defining the path loss model for aerial users above $22.5 \mathrm{~m}$ AGL. The general expression is as follows:

$$
P L=C+D \times \log _{10}\left(d_{3 D}\right)+20 \log _{10}\left(f_{c}\right),
$$

where $C$ and $D$ are scenario (i.e., environment and LOS/NLOS link) and altitude-dependent constants (for the values, refer to 3GPP [3] and [2]); $d_{3 D}$ is the 3D distance between the nodes; $f_{c}$ is the carrier frequency. For example, PL for UAVs flying higher than $22.5 \mathrm{~m}$ and with a LOS connection is expressed as:

$$
P L=28.0+22 \times \log _{10}\left(d_{3 D}\right)+20 \log _{10}\left(f_{c}\right) .
$$

An example of the resulting interference map is shown in Figure 2.

\subsection{Permissible interference for satellite services}

We used the methodology proposed in [13] and [11], where a method to estimate the level of maximum permissible interference at the input of FSS earth station is described. Permissible interference power in the reference bandwidth is the power level to be exceeded for no more than $p$ percent of the time at the receiver input of a victim station(in this case, a VSAT station). Interference

Table 2: Used satellites

\begin{tabular}{lcc}
\hline Name & Azimuth [deg] & Elevation [deg] \\
\hline Intelsat 20 & 110.9 & 7.3 \\
Intelsat 906 & 114.5 & 10.1 \\
YAMAL 202 & 128.5 & 18.6 \\
NSS 7 & 210.7 & 27.3 \\
Eutelsat 5W & 192.4 & 31 \\
\hline
\end{tabular}

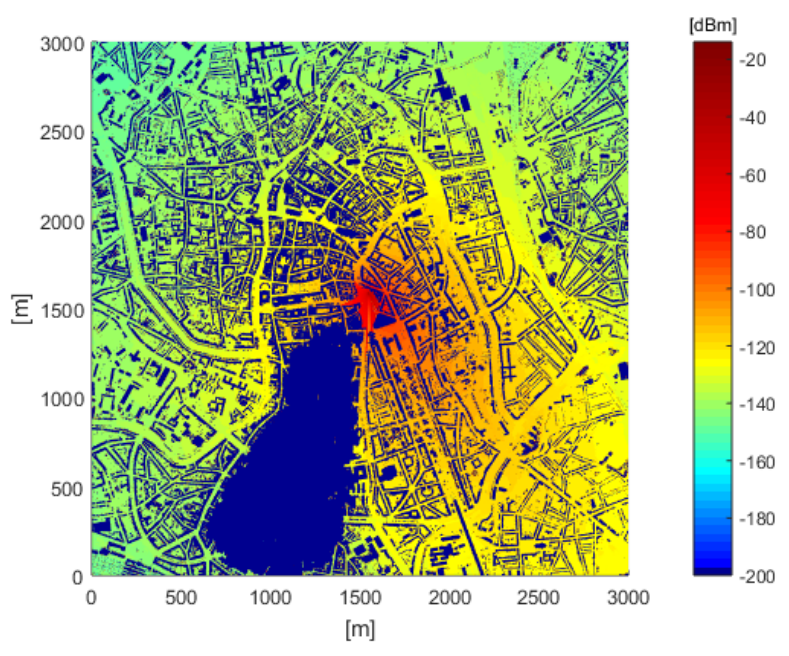

Figure 2: The interference map of the city centre of Ghent

between a VSAT station and a UAV can be considered as negligible when the interference power level does not exceed the maximum permissible level of interference for $20 \%$ of the time. However, in some cases, it is necessary to compare the received interference power and the minimum permissible power received from the UAV, at which interference is negligible for two time percentages on the one hand equal to $20 \%$ of the time $p_{1}$, and on the other, a low percentage of the time $(0.5 \%)$ designated $p_{2}$.

The long-term ( $p_{1}=20 \%$ of the time) maximum permissible interference level is given by

$$
P_{r}(20 \%)=10 \log \left(k T_{r} B\right)+J+W,
$$

where $k$ is Boltzmann's constant $\left(1.38 \cdot 10^{-23}(J / K)\right)$, $J$ is a ratio of the permissible long-term interfering power from any one interfering source to the thermal noise power in the FSS system and $W$ is a thermal noise equivalence factor $(\mathrm{dB})$ for interfering emissions in the reference bandwidth. We set $J=-10$ and $W=0$, according to the recommendations in [13].

Another threshold of $p_{2}=0.5 \%$ is chosen to reflect significantly enhanced (small percentages of the time) interference conditions, for which interference contributions from all interfering sources may be assumed to occur non-simultaneously. It is given by:

$$
P_{r}(0.5 \%)=10 \log \left(k T_{r} B\right)+10 \log \left(10^{M_{s} / 10}-1\right)+N_{L}-W,
$$

where $M_{s}=37$ is fade margin of link (see [11], Note 3); $N_{L}=0$ is link noise contribution (see [11], Note 4).

Correspondent permissible levels are $P_{r}(20 \%)=-118.6 \mathrm{dBm}$ and $P_{r}(0.5 \%)=-110.9 \mathrm{dBm}$.

\section{SIMULATION RESULTS}

The generated interference maps were used to obtain the no-fly zones, i.e. the areas where a flying UAV could affect VSAT operation. Examples of these regions are shown in Figure 3 for $P_{r}(0.5 \%)$ (top) and $P_{r}(20 \%) \mathrm{dBm}$ (bottom). For this simulation, the UAV was located on the same altitude as the considered VSAT receiving signal from YAMAL 202 satellite. The minimum separation distance varies 
between $300 \mathrm{~m}$ and $200 \mathrm{~m}$ for $P_{r}(0.5 \%)$ and $P_{r}(20 \%)$, respectively. The maximum separation distance is ranging from $700 \mathrm{~m}$ to $1.2 \mathrm{~km}$ for the two considered interference levels. Note that this is an optimistic estimation since the UAV altitude is low. This situation is similar to co-existence of the VSAT and ground users (higher PL). Note that these results are environment specific and cannot be used for drawing the generalized UAV usage recommendations.

Even for this optimistic estimation the no-fly zone for one VSAT is already of a significant size. Considering that a UAV will be exposed to multiple VSAT stations one could see that the no-fly zones would pose a large problem for UAV deployment in areas with a high density of VSAT deployments, which is the case in equatorial areas for example. We can see that interference protection and mitigation techniques such as smart frequency planning will be necessary. For example [6] showed that a frequency shift of $28 \mathrm{MHz}$ already provides an extra 14 to $20 \mathrm{~dB}$ extra protection.
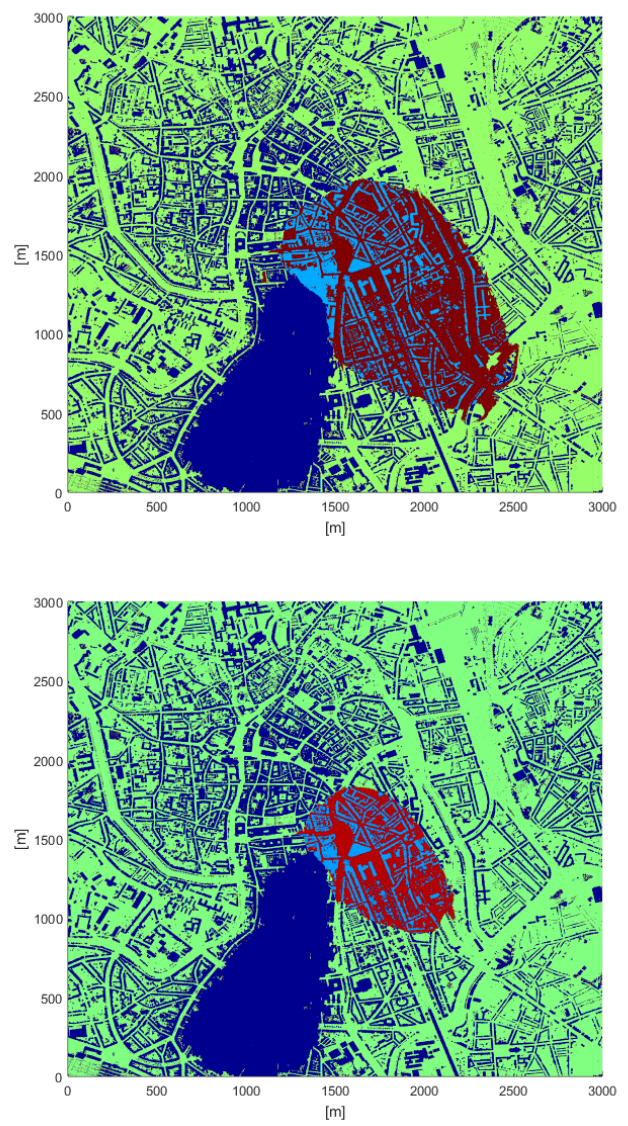

Figure 3: Map of Ghent fly zones, $P_{r}(0.5 \%)$ (top) and $P_{r}(20 \%)$ dBm (bottom). Dark blue represents intersection with terrain or buildings, dark red represents a no-fly zone and green means no restrictions for flying drones.

\subsection{Stochastic Interference Estimation}

Next, let us statistically analyze the interference levels depending on the angle $\Theta$ and distance $d_{3 D}$ between the interfering UAV and the main axis of the VSAT antenna. Figure 4 demonstrates the obtained results. The angle and distance-dependent interference levels $I\left(\Theta, d_{3 D}\right)$ are shown in the top figure. The angle dependence is smooth due to the used VSAT antenna patterns (as it is recommended in [15] for obtaining the generalized results, the side-lobes envelope is used rather than the exact antenna pattern). Irregular behavior of the interference levels for small $\left(<10^{\circ}\right)$ and large $\left(>165^{\circ}\right)$ angles can be explained by the environment influence (obstructed LOS, high PL).

Figure 4 (bottom) shows the average interference levels created by UAV flying on- and -off axis, on-axis meaning the UAV is positioned inside the main beam of VSAT antenna pattern. The simulations show that, on average, UAV does not interfere with VSATs located farther than $500 \mathrm{~m}$, in the case when the UAV is located behind the satellite antenna (off-axis). In our simulations, when the drone is flying in the main lobe of a VSAT antenna (onaxis), the averaged interference level is always higher than the permissible levels. Due to the limitation of the altitude of the UAV's, the simulation only covered distances up to $1500 \mathrm{~m}$ on the on-axis. By fitting the results of the on-axis, we found that the separation distance of $3500 \mathrm{~m}$ would be needed to achieve $P_{r}(0.5 \%)$, however, the corresponding UAV altitude is around $1800 \mathrm{~m}$ (for YAMAL 202), which is much higher than the typical UAV deployment heights.
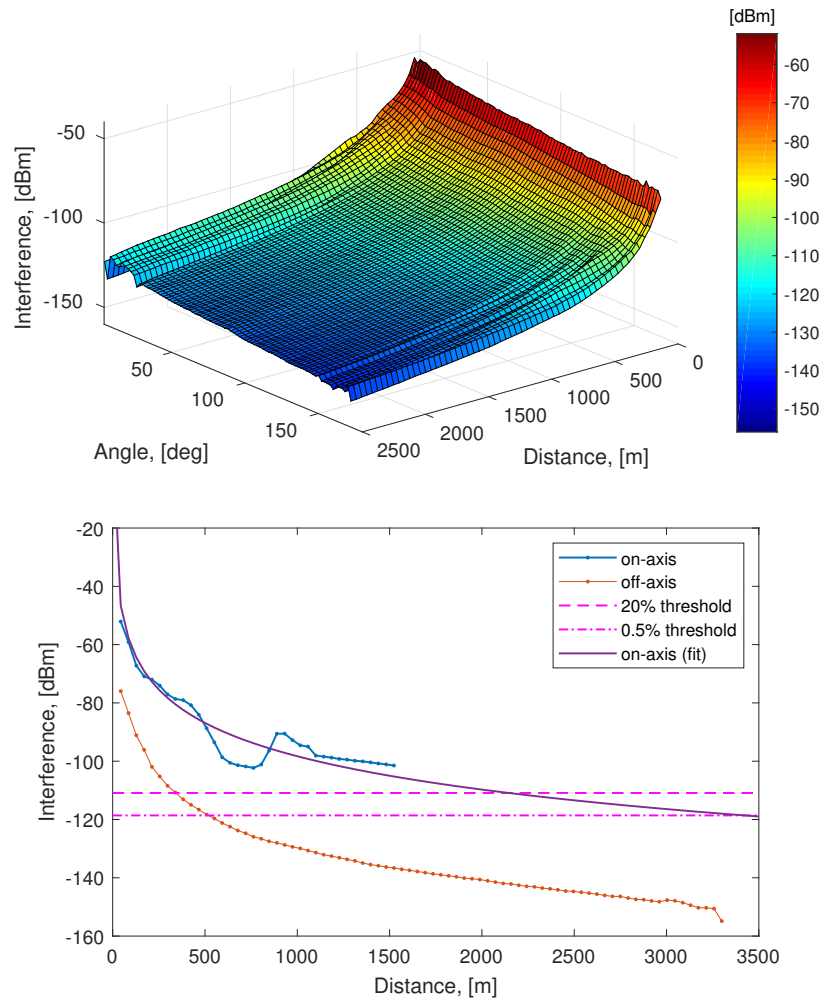

Figure 4: Averaged Interference levels received by VSATs 

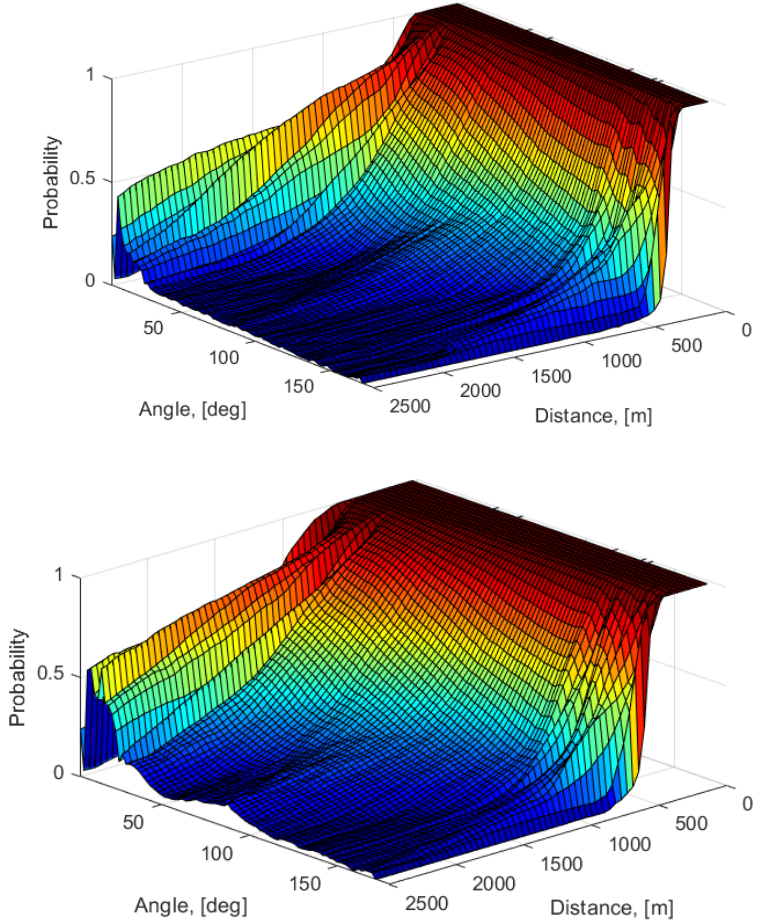

Figure 5: Probability exceeding the maximum permissible interference levels (top: $P_{r}(20 \%)$ and bottom: $P_{r}(0.5 \%)$ )

\subsection{Probability of exceeding the permissible interference levels}

Figure 5 shows the angle-distance dependent behavior of the probability of exceeding the maximum permissible interference levels. Note that on-axis case is not included in this plot due to the large distances needed to achieve the targeted interference levels. We observe $100 \%$ probability for an UAV interfering with VSAT if the direct distance between them is less than $170 \mathrm{~m}$ and $300 \mathrm{~m}$ (independent on the angle) for $P_{r}(0.5 \%)$ and $P_{r}(20 \%)$, respectively. Interestingly, the UAV positions with the angles smaller than $25^{\circ}$ are showing higher interfering levels. This might be explained by both environment impact and the VSAT antenna gain. Relatively low interference probability is expected for the UAV locations with $\Theta>25^{\circ}$ and $d_{3 D}>1000 \mathrm{~m}$.

Figure 6 shows the minimum separation distances defined as

- distance where the interference is strictly smaller than the permissible levels $\mathbf{P}\left(I<P_{r}\right)=1$;

- distance where the averaged interference $I$ is smaller than the permissible levels $\bar{I} \leq P_{r}$.

Depending on the definition, the separation distance is varying between $500 \mathrm{~m}$ (only long-term averaged interference) and $4000 \mathrm{~m}$ (no short-term interference $\mathbf{P}\left(I<P_{r}(0.5 \%)\right)=1$ )

\section{CONCLUSIONS}

This work presents results of a large scale simulation campaign to assess the coexistence requirements for satellite systems in C-band

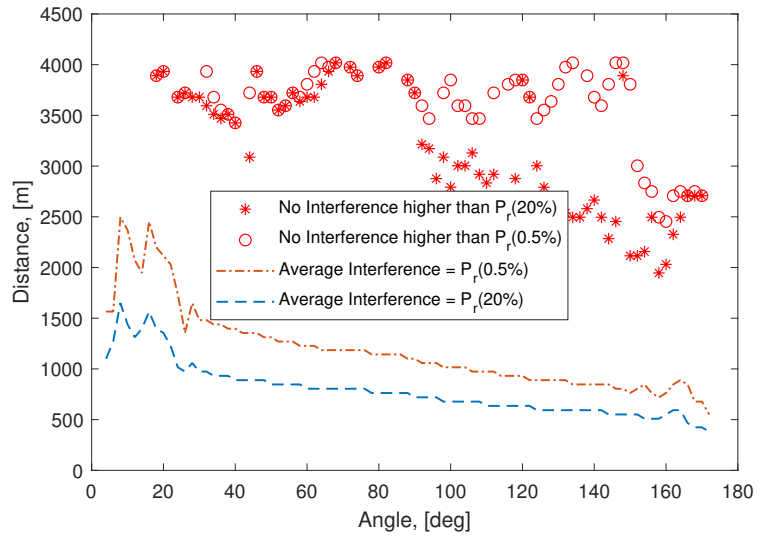

Figure 6: Angular dependence of the safe separation distance

and $4 \mathrm{G}$ or $5 \mathrm{G}$ cellular connected UAVs. Proper separation distances for co-frequency coexistence conditions have been identified in different realistic propagation conditions simulated by means of a real 3D map of a European city.

The used approach can be applied also in other scenarios, and will provide useful information to regulatory bodies in defining appropriate rules for coexistence between satellite and UAVs connected to cellular networks. It has been shown that the interference created by UAVs is significant and poses serious danger to existing satellite services. Mobile operators will have to guarantee adequate protection for the satellite systems, by means of interference mitigation techniques such as frequency separation, radiation diagram optimization, active antenna systems, etc.

Possible solutions include (but are not restricted to)

- Frequency separation: in [6], it was shown that a frequency offset of $28 \mathrm{MHz}$ can bring a significant improvement of the experienced interference due to an additional protection in the range of 14 to $20 \mathrm{~dB}$;

- More advanced antenna systems on the UAV side can lower the created interference (similar conclusion was made in [5] and [18] for other UAV applications);

- Beamforming on the BS side (for the interference in downlink) as shown in [7].

\section{ACKNOWLEDGMENTS}

This research was funded by the imec.icon project 5GUARDS and the Research Foundation Flanders (FWO), project no. S003817N (OmniDrone).

\section{REFERENCES}

[1] [n. d.]. Agentschap voor Geografische Informatie Vlaanderen: Standaardproducten Digitaal Hoogtemodel Vlaanderen II. https: //download.agiv.be/Producten/Detail?id=966\&title=Standaardproducten

Digitaal_Hoogtemodel_Vlaanderen_II. Last accessed on 2018-07-26.

[2] 3GPP. 2017. Enhanced LTE support for aerial vehicles. Technical Report 36.777. https://portal.3gpp.org/desktopmodules/Specifications/ SpecificationDetails.aspx? specificationId $=3231$

[3] 3GPP. 2017. Study on channel model for frequencies from 0.5 to $100 \mathrm{GHz}$. Technical Report 38.901. https://portal.3gpp.org/desktopmodules/Specifications/ 
SpecificationDetails.aspx?specificationId $=3231$

[4] 3GPP. 2018. NR; Base Station (BS) radio transmission and reception. Technical Specification 38.104. https://portal.3gpp.org/desktopmodules/Specifications/ SpecificationDetails.aspx? specificationId $=3202$

[5] M. M. Azari, F. Rosas, A. Chiumento, A. Ligata, and S. Pollin. 2018. Uplink performance analysis of a drone cell in a random field of ground interferers. In 2018 IEEE Wireless Communications and Networking Conference (WCNC). 1-6. https://doi.org/10.1109/WCNC.2018.8377426

[6] C. Carciofi, P. Grazioso, V. Petrini, E. Spina, D. Massimi, G. De Sipio, E. Scognamiglio, V. Sorrentino, A. Casagni, L. Guoyue, Z. Lai, and R. Rudd. 2018. Cochannel and adjacent-channel coexistence between LTE-TDD and VSAT DVB-S in C-band: experimental campaign on consumer VSAT receivers. In 2018 IEEE 29th Annual International Symposium on Personal, Indoor and Mobile Radio Communications (PIMRC). 1-5. https://doi.org/10.1109/PIMRC.2018.8580923

[7] A. Colpaert, E. Vinogradov, and S. Pollin. 2018. Aerial Coverage Analysis of Cellular Systems at LTE and mmWave Frequencies Using 3D City Models. Sensors 18(12):4311 (2018)

[8] ECC. 2007. Compatibility studies in the band 3400-3800 MHz between Broadband Wireless access (BWA) systems and other services. Report.

[9] ECC. 2013. Least Restrictive Technical Conditions suitable for Mobile/Fixed Communication Networks (MFCN), including IMT, in the frequency bands 3400-3600 $\mathrm{MHz}$ and $3600-3800 \mathrm{MHz}$. Report.

[10] ECC. 2016. Operational guidelines for spectrum sharing to support the implementation of the current ECC framework in the $3600-3800 \mathrm{MHz}$ range. Report.

[11] ITU-R. 1993. Determination of the interference potential between earth stations of the fixed-satellite service and stations in the fixed service. Recommendation SF.1006. https://www.itu.int/rec/R-REC-SF.1006/en

[12] ITU-R. 2007. Sharing studies between IMTAdvanced systems and geostationary satellite networks in the fixed-satellite service in the $3400-4200$ and 4500-4 800
MHz frequency bands. Report M.2109.

[13] ITU-R. 2010. DStudies on compatibility of broadband wireless access systems and fixed-satellite service networks in the 3 400-4 $200 \mathrm{MHz}$ band. Report S.2199. https://www.itu.int/pub/R-REP-S.2199

[14] ITU-R. 2010. Methodology on the modelling of earth station antenna gain in the region of the antenna main-lobe and the transition region between the minimum angle of the reference antenna pattern and the main-lobe. Recommendation S.2196. http://www.itu.int/pub/R-REP-S.2196/en

[15] ITU-R. 2010. Reference radiation pattern of earth station antennas in the fixedsatellite service for use in coordination and interference assessment in the frequency range from 2 to $31 \mathrm{GHz}$. Recommendation S.465. https://www.itu.int/rec/R-REC-S. 465-6-201001-I/en

[16] ITU-R. 2014. Reference radiation patterns of omnidirectional, sectoral and other antennas for the fixed and mobile service for use in sharing studies in the frequency range from $400 \mathrm{MHz}$ to about $70 \mathrm{GHz}$. Recommendation F.1336. https://www.itu. int/rec/R-REC-F.1336/en

[17] B. Van Den Bergh, A. Chiumento, and S. Pollin. 2016. LTE in the sky: trading off propagation benefits with interference costs for aerial nodes. IEEE Communications Magazine 54, 5 (May 2016), 44-50. https://doi.org/10.1109/MCOM.2016. 7470934

[18] E. Vinogradov, D. A. Kovalev, and S. Pollin. 2018. Simulation and Detection Performance Evaluation of a UAV-mounted Passive Radar. In 2018 IEEE 29th Annual International Symposium on Personal, Indoor and Mobile Radio Communications (PIMRC). 1185-1191. https://doi.org/10.1109/PIMRC.2018.8580940

[19] E. Vinogradov, H. Sallouha, S. De Bast, M. M. Azari, and S. Pollin. 2018. Tutorial on UAVs: A Blue Sky View on Wireless Communication. Journal of Mobile Multimedia 14, 4 (October 2018), 395-468. https://doi.org/10.13052/jmm1550- 4646.1443 